\title{
Suppression of gravitational radiation
}

\author{
W.B.Bonnor and M.S.Piper \\ School of Mathematical Sciences, \\ Queen Mary and Westfield College, \\ Mile End Road, \\ London. \\ E1 4NS
}

May 25, 2017

\begin{abstract}
We consider a burst of quadrupole gravitational radiation in the presence of a large static mass $M$ situated at its source. Some of the radiation is back-scattered off the static field of the large mass, forming a wave tail. After the burst, the tail is a pure incoming wave, carrying energy back towards the source. We calculate this energy, and, in a numerical example, compare it with the outgoing wave energy. If $M$ is sufficiently large the incoming energy can equal the outgoing energy, indicating that the primary outgoing wave is completely suppressed.
\end{abstract}

\section{Introduction}

It has been known for many years that gravitational waves have tails [1][2][3]. (For a good literature survey see [4]). Tails are now recognised as factors in the planned observational detection of the waves [5][14][15]. The simplest sort of tail results from the back-scattering of an outgoing wave by the static field of the source, or of other bodies. Let us suppose for simplicity that there is an outgoing quadrupole wave that lasts for a finite time; when it ceases the tail persists, and as shown by Hunter and Rotenberg [3], it is then a pure incoming quadrupole wave. As such it carries energy, and this is what we calculate in this paper. The fate of this energy is not certain. It might be reabsorbed by the source, it might pass through the centre and become outgoing, or it might even form a singularity as suggested by the numerical work of Abraham and Evans[6][7]. In this paper we do not consider these possibilities, but merely study the relative energies of the primary outgoing wave and the tail.

The incoming energy carried by the tail must, of course, decrease the outgoing wave energy, as given, for example, by the quadrupole formula. The 
question therefore arises whether the calculated incoming energy could be greater than the outgoing, which would presumably mean that the static fields of the source and its neighbours suppress the waves entirely. From the theoretical point of view there seems no objection to this, since a similar suppression can occur of a light wave by a black hole. Indeed the suppression was investigated some years ago from a different point of view by Kundu [8], and his work caused some debate [9][10]. The conclusion seemed to be that a suppressive effect existed but would be counteracted by other phenomena, and would have no astrophysical importance [11].

The plan of the paper is as follows. We describe the metric and approximation method in Sections 2 and 3, and follow these with the linear approximation to the outgoing quadrupole wave in Section 4. We give the formulae for the wave tail in Section 5, and for the energy it carries after the vibration ceases in Section 6. Section 7 contains numerical calculations of the energies carried outward by the quadrupole wave and inward by its tail for a specified pulse. Conclusions are expressed in Section 8.

\section{Metric}

When dealing with outgoing waves we shall use the axially symmetric metric of Bondi [12]:

$$
d s^{2}=-r^{2}\left(B d \theta^{2}+B^{-1} \sin ^{2} \theta d \phi^{2}\right)+D d u^{2}+2 F d r d u+2 r G d \theta d u
$$

where $B, D, F, G$ are functions of $r, \theta$ and $u$. $u$ is a retarded time coordinate and may be thought of as ordinary time $t$ at the source, which is represented by a singularity at the origin. The Schwarzschild solution in the coordinates of (1) is

$$
d s^{2}=-r^{2}\left(d \theta^{2}+\sin ^{2} \theta d \phi^{2}\right)+\left(1-2 M r^{-1}\right) d u^{2}+2 d r d u .
$$

We shall also be studying incoming waves and for these we use the Bondi metric in the form

$$
d s^{2}=-r^{2}\left(B d \theta^{2}+B^{-1} \sin ^{2} \theta d \phi^{2}\right)+D d v^{2}-2 F d r d v+2 r G d \theta d v
$$

with $B, D, F, G$ now functions of $r, \theta$ and $v$, and $v$ an advanced time coordinate. In this metric the Schwarzschild solution is

$$
d s^{2}=-r^{2}\left(d \theta^{2}+\sin ^{2} \theta d \phi^{2}\right)+\left(1-2 M r^{-1}\right) d v^{2}-2 d r d v
$$

In these metrics the ranges are

$$
r>0, \quad 0 \leq \theta \leq \pi, \quad 0 \leq \phi \leq 2 \pi, \quad-\infty<u, v<\infty
$$




\section{The approximation method}

We use an adaptation of the double series method. We suppose that the solution we are looking for can be expanded in a doubly infinite power series in $M$, a central spherical mass, and a parameter $Q$ characterising the primary quadrupole moment of the vibrating source. The actual quadrupole moment at time $t$ is $Q h(t)$, and $h$ is zero except in $t_{1}<t<t_{2}$. Therefore we envisage an expansion of the $g_{i k}$ of the form

$$
g_{i k}=\sum_{p=0}^{\infty} \sum_{s=0}^{\infty} M^{p} Q^{s} \stackrel{(p s)}{g},
$$

the $\stackrel{(p s)}{g}$ ik being independent of $M$ and $Q$.

If we substitute (5) into the vacuum field equations

$$
R_{i k}=0
$$

and consider the coefficient of $M^{p} Q^{s}$ we find seven equations of the form

$$
\Phi_{l m}\left(\stackrel{(p s)}{g_{i k}}\right)=\Psi\left(\stackrel{(q r)}{g_{i k}}\right),
$$

where the left-hand side is linear in the $\stackrel{(p s)}{g}_{i k}$ (and their derivatives), and the right-hand side consists of terms non-linear in the $\stackrel{(q r)}{g}$ ik and their derivatives, with $q \leq p, r \leq s$ and $q+r<p+s$. This is called the ( $p s)$ approximation. We can introduce this notation into the metric coefficients of (1), for example:

$$
-g_{22}=r^{2} B=r^{2}\left[1+\sum_{p=0}^{\infty} \sum_{s=0}^{\infty} M^{p} Q^{s} \stackrel{(p s)}{B}\right]
$$

(00)

where $B$ is zero. In this paper we shall go in detail only as far as the (11) approximation; this will express the interaction of the central mass and the quadrupole oscillations, which describes the simplest wave tail. To calculate the energies transmitted by the quadrupole wave and its tail we shall need formulae from the (02) and (22) approximations respectively which are known from previous work, namely eqns (12) and (29).

\footnotetext{
${ }^{1}$ By primary quadrupole moment is meant the one inserted at the linear approximation, generating waves against a Minkowski background, and entering the usual quadrupole formula. Quadrupole waves appear in higher approximations and one might regard them as arising from higher quadrupole moments, though we do not do this here. Hereafter we drop the prefix 'primary', and refer simply to the quadrupole moment.
} 


\section{The linear approximation}

We write down the solution, in the coordinates of (1), of the linearised form of (6) (i.e. (7) with $\Psi=0$ ) corresponding to a outgoing quadrupole wave [1].

$$
\begin{aligned}
\stackrel{(01)}{B} & =\frac{1}{2} \sin ^{2} \theta\left(r^{-1} \ddot{h}+r^{-3} h\right), \\
\stackrel{(01)}{D} & =-P_{2}\left(2 r^{-1} \ddot{h}+2 r^{-2} \dot{h}+r^{-3} h\right), \\
\stackrel{(01)}{F} & =0, \\
\stackrel{(01)}{G} & =\frac{1}{2} \sin \theta \cos \theta\left(-2 r^{-1} \ddot{h}+4 r^{-2} \dot{h}+3 r^{-3} h\right),
\end{aligned}
$$

where $P_{2}(\cos \theta)$ is the Legendre polynomial and an overdot means differentiation with respect to $u$. $h(u)$ is zero except for $u_{1}<u<u_{2}$, where $u_{i}=t_{i}$, and represents the quadrupole oscillation. We write $Q h$ for the quadrupole moment of the source. We shall assume $h$ is of class $C^{3}$ for $u>u_{1}$ so that we can perform the necessary calculations on the tail in Section 7.

The energy carried outward by this oscillation, calculated by the usual quadrupole formula or as a loss of mass as in [1], is

$$
E_{\text {out }}=\frac{Q^{2}}{30} \int_{u_{1}}^{u_{2}} \dddot{h}^{2} d u \text {. }
$$

\section{The wave tail}

The tail comes from the interaction of the quadrupole wave with the Schwarzschild metric. These are respectively the (01) and the (10) approximations, given by (8)-(11) and (2). Inserting them into the right-hand side of (7) we have seven differential equations for the $\stackrel{(11)}{g}_{i k}$, of which the solution is [3][13]. . ?

$$
\begin{aligned}
\stackrel{(11)}{B} & =\frac{1}{8} \sin ^{2} \theta\left(2 r^{-3} \dot{h}+10 r^{-4} h+r^{-1} \frac{\partial^{2} H}{\partial r^{2}}-4 r^{-2} \frac{\partial H}{\partial r}+4 r^{-3} H\right), \\
\stackrel{(11)}{D} & =-\frac{1}{2} P_{2}\left(r^{-3} \dot{h}+2 r^{-4} h+2 r^{-3} H\right), \\
\stackrel{(11)}{F} & =0 \\
\stackrel{(11)}{G} & =\frac{1}{8} \sin 2 \theta\left(3 r^{-3} \dot{h}+8 r^{-4} h-2 r^{-2} \frac{\partial H}{\partial r}+6 r^{-3} H\right),
\end{aligned}
$$

where $\mathrm{H}(\mathrm{r}, \mathrm{u})$ is a tail function defined by

$$
H=-2 \frac{\partial}{\partial u} \int_{-\infty}^{u} \frac{h(\xi) d \xi}{(u+2 r-\xi)}
$$

\footnotetext{
${ }^{2}$ In the previous work cited the notation was different and the tail approximation was denoted (22) instead of (11).
} 
From the definition of $H$ we find

$$
\frac{\partial H}{\partial r}-2 \frac{\partial H}{\partial u}=2 r^{-1} \dot{h}
$$

now $\dot{h}=0$ after the end of the quadrupole oscillation so

$$
H(r, u)=H(u+2 r), u>u_{2} .
$$

In the flat background spacetime $u+2 r$ is the advanced time coordinate (hereafter denoted by $v$ ) so for $u>u_{2}$ the tail function $H$ is an incoming wave. From (17)

$$
H=-\frac{h(u)}{r}+2 \int_{-\infty}^{u} \frac{h(\xi) d \xi}{(u+2 r-\xi)^{2}}
$$

so as $h$ as well as $\dot{h}$ is zero for $u>u_{2}$, we have for the tail function after the vibration has finished

$$
H_{0}=2 \int_{-\infty}^{u} \frac{h(\xi) d \xi}{(u+2 r-\xi)^{2}}, u>u_{2} .
$$

Since $H_{0}$ is a function of $v=u+2 r$ we can write (13)-(16) for $u>u_{2}$ as

$$
\begin{aligned}
\stackrel{(11)}{B} & =\frac{1}{2} \sin ^{2} \theta\left(r^{-1} H_{0}^{\prime \prime}-2 r^{-2} H_{0}^{\prime}+r^{-3} H_{0}\right), \\
\stackrel{(11)}{D} & =-P_{2} r^{-3} H_{0}, \\
\stackrel{(11)}{F} & =0, \\
\stackrel{(11)}{G} & =\frac{1}{4} \sin 2 \theta\left(-2 r^{-2} H_{0}^{\prime}+3 r^{-3} H_{0}\right),
\end{aligned}
$$

where $/$ means $d / d v$. This is the linear approximation for an incoming quadrupole wave in outgoing Bondi coordinates. It was given in [3], eqn (7.9) with $s=2$ and $\stackrel{2}{f}=-H_{0}$. As $v=u+2 r$ and $u>u_{2}, r>0,(20)-(23)$ apply for

$$
v>u_{2},
$$

$u_{2}$ being, of course, a constant.

In order to calculate the energy carried by this incoming wave we write it in incoming Bondi coordinates, eqn (3). This too was done in [3] eqn (A25), and the result is

$$
\begin{aligned}
\stackrel{(11)}{B} & =\frac{1}{2} \sin ^{2} \theta\left(r^{-1} H_{0}^{\prime \prime}+r^{-3} H_{0}\right), \\
\stackrel{(11)}{D} & =-P_{2}\left(2 r^{-1} H_{0}^{\prime \prime}-2 r^{-2} H_{0}^{\prime}+r^{-3} H_{0}\right), \\
\stackrel{(11)}{F} & =0, \\
\stackrel{(11)}{G} & =\frac{1}{2} \sin \theta \cos \theta\left(2 r^{-1} H_{0}^{\prime \prime}+4 r^{-2} H_{0}^{\prime}-3 r^{-3} H_{0}\right) .
\end{aligned}
$$




\section{$6 \quad$ Energy carried by the wave tail}

In the case of outgoing waves the energy carried is given by the loss of mass of the source. The simplest approximation in which it appears is that of the quadrupole-quadrupole interaction, so that in our notation of Section 3 it is of order $Q^{2}$. This approximation was completely solved in [3], and the loss of mass, given by eqn (12), agreed with the ordinary quadrupole formula.

The incoming waves described by (25)-(28) are of order $M Q$, but they have quadrupole form, and one can adapt the calculations of [3] to solve the $M^{2} Q^{2}$ approximation in the incoming case. In fact the solution has exactly the same form as that of [3] 3., except for changes of sign. (See Appendix 1). The result is that the energy flowing inward after the vibration ceases is

$$
E_{\text {in }}=\frac{M^{2} Q^{2}}{30} \int_{u_{2}}^{\infty} H_{0}^{\prime \prime \prime 2} d v
$$

$H_{0}$ being defined by (19). The lower limit of the integral arises from (24).

\section{$7 \quad$ An example}

To get a feeling for the magnitudes involved we construct an artificial example in which $h(u)$ is a single pulse given by

$$
h(u)=(1-u)^{5},-1<u \leq 1 ; h(u)=0,|u| \geq 1 .
$$

This has been chosen to make the tail function $H$ reasonably simple. Although $h$ is discontinuous at $u=-1$ it is very smooth at $u=1$ which is sufficient to give an $H_{0}$ which can be used in (29) if we put $u_{2}=1$. In this way we can calculate the energy carried inward by the tail after the end $u=u_{2}$ of the vibration.

First we calculate the energy $E_{\text {out }}$ carried outward by the pulse $h$ according to (12):

$$
E_{\text {out }}=\frac{Q^{2}}{30} \int_{-1}^{1} \dddot{h}^{2} d u=768 Q^{2} .
$$

where we have inserted the form (30) for $h$.

From (19) we have for $H_{0}(v), v \geq 1$ :

$$
H_{0}(v)=2 \int_{-1}^{1} \frac{(1-\xi)^{5}}{(v-\xi)^{2}} d \xi, v>1
$$

whence, after a fairly long but straightforward calculation,

$$
H^{\prime \prime \prime}=16(1+v)^{-4}\left(64+30 v-70 v^{2}-90 v^{3}-30 v^{4}\right)+240(v-1)[\ln (v+1)-\ln (v-1)] .
$$

\footnotetext{
${ }^{3}$ There described as the quadrupole-quadrupole approximation
} 
To find $E_{\text {in }}$ we have to calculate the integral (29). This can be evaluated exactly using REDUCE. The result is

$$
E_{\text {in }}=28.2 M^{2} Q^{2}
$$

to three significant figures.

These expressions for the inward and outward energies are in relativistic units. We need to find their ratio when they are expressed in units of customary dimensions. Denoting them in such units by $W_{\text {in }}$ and $W_{\text {out }}$ we show in Appendix 2 that the ratio is

$$
\frac{W_{\text {in }}}{W_{\text {out }}}=\frac{G^{2} m^{2}}{c^{6}} \frac{\int_{1}^{\infty} H_{0}^{\prime \prime \prime} d v}{\int_{-1}^{1} h^{\prime \prime \prime} d u} .
$$

Using the values of the integrals calculated above we find

$$
\frac{W_{\text {in }}}{W_{\text {out }}} \sim 2 \times 10^{-79} \mathrm{~m}^{2},
$$

where $m$ is in grams. To render $W_{\text {in }}$ comparable with $W_{\text {out }}$ the central mass would have to be that of a large black hole.

\section{Conclusion}

We have shown that in the presence of a sufficiently large mass the incoming energy of the tail can be comparable or even equal to that of the outgoing wave pulse. This raises the possibility that a pulse of radiation from, say, a coalescing binary might be significantly reduced, or even suppressed, if the static gravitational fields in its neighbourhood are great enough.

It must be emphasised that our calculations are not intended as a serious astrophysical model. We have treated the neighbours of the source as a single static spherical mass at the position of the source, and our numerical calculations are for one very special shape of pulse. Moreover, we have considered the tail only after the primary pulse has ceased. Nevertheless, we believe that the incoming tail energy should be taken carefully into account in gravitational wave experiments.

\section{Appendix 1}

In this Appendix we derive formulae relating to the incoming wave-tail energy referred to in Section 6. For this we make use of the outgoing quadrupolequadrupole solution given by equations (3.7)-(3.11) in [3]. To obtain the complete $M^{2} Q^{2}$ solution in the incoming case we simply make the coordinate transformation

$$
u=-v
$$


and the identification

$$
h=H_{0}
$$

with $r, \theta$ and $\phi$ remaining unchanged and where $h$ is written as $\stackrel{2}{h}$ in [3]. The only part of the transformation that is not entirely straightforward is the way in which $\stackrel{(24)}{Y}$ of [3] transforms to its equivalent $Y$ here. The former is defined by

$$
\frac{d \stackrel{(24)}{Y}}{d u}=\dddot{h}^{2}
$$

where and overdot denotes differentiation with respect to $u$, and so $Y$ must satisfy

$$
-\frac{d Y}{d v}=H_{0}^{\prime \prime \prime 2}
$$

where ' denotes differentiation with respect to $v$. Integrating with respect to $v$ gives

$$
Y=-\int_{u_{2}}^{\infty} H_{0}^{\prime \prime \prime 2} d v
$$

The transformation (37), used on (3.7)-(3.11) of [3] then gives:

$$
\begin{aligned}
\stackrel{(22)}{D}= & {\left[\left(\frac{3}{5}-3 s^{2}+\frac{21}{8} s^{4}\right) H_{0}^{\prime} H_{0}^{\mathrm{IV}}+\left(\frac{12}{5}-9 s^{2}+\frac{27}{4} s^{4}\right) H_{0}^{\prime \prime} H_{0}^{\prime \prime \prime}+\frac{1}{15} Y\right] r^{-1} } \\
& +\left[\left(-2+10 s^{2}-\frac{35}{4} s^{4}\right) H_{0}^{\prime} H_{0}^{\prime \prime \prime}+\left(-2+9 s^{2}-\frac{15}{2} s^{4}\right) H_{0}^{\prime \prime 2}\right] r^{-2} \\
& +\left(\frac{9}{2}-\frac{47}{2} s^{2}+\frac{331}{16} s^{4}\right) H_{0}^{\prime} H_{0}^{\prime \prime} r^{-3}+\left[\left(\frac{3}{2} s^{2}-\frac{27}{16} s^{4}\right) H_{0} H_{0}^{\prime \prime}\right. \\
& \left.+\left(-\frac{15}{4}+\frac{71}{4} s^{2}-\frac{493}{32} s^{4}\right) H_{0}^{\prime 2}\right] r^{-4}+\left(\frac{3}{2}-6 s^{2}+\frac{81}{16} s^{4}\right) H_{0}^{\prime} H_{0} r^{-5} \\
& +\left(\frac{1}{2}-3 s^{2}+\frac{21}{8} s^{4}\right) H_{0}^{2} r^{-6} \\
\stackrel{(22)}{F}=^{4}= & s^{4}\left[-\frac{1}{32} H_{0}^{\prime \prime 2} r^{-2}-\frac{3}{32} H_{0} H_{0}^{\prime \prime} r^{-4}-\frac{3}{32} H_{0}^{2} r^{-6}\right] \\
\stackrel{(22)}{G}= & {\left[\left(-\frac{3}{10} c s+\frac{21}{40} c s^{3}\right) H_{0}^{\prime} H_{0}^{\mathrm{IV}}+\left(-\frac{6}{5} c s+\frac{27}{20} c s^{3}\right) H_{0}^{\prime \prime} H_{0}^{\prime \prime \prime}-\left(\frac{1}{30} c s+\frac{1}{40} c s^{3}\right) Y\right] r^{-1} } \\
& +\left[\left(-2 c s+\frac{7}{2} c s^{3}\right) H_{0}^{\prime} H_{0}^{\prime \prime \prime}+\left(-2 c s+\frac{23}{8} c s^{3}\right) H_{0}^{\prime \prime 2}\right] r^{-2} \\
& +\left(\frac{27}{4} c s-\frac{185}{16} c s^{3}\right) H_{0}^{\prime} H_{0}^{\prime \prime} r^{-3}+\left[\frac{1}{8} c s^{3} H_{0} H_{0}^{\prime \prime}+\left(-\frac{15}{2} c s+\frac{105}{8} c s^{3}\right) H_{0}^{\prime 2}\right] r^{-4} \\
& +\left(\frac{15}{4} c s-\frac{107}{16} c s^{3}\right) H_{0} H_{0}^{\prime} r^{-5}+\left(\frac{3}{2} c s-\frac{21}{8} c s^{3}\right) H_{0}^{2} r^{-6} \\
= & {\left[\left(-\frac{3}{20} s^{2}+\frac{7}{40} s^{4}\right) H_{0}^{\prime} H_{0}^{\mathrm{IV}}+\left(-\frac{3}{5} s^{2}+\frac{9}{20} s^{4}\right) H_{0}^{\prime \prime} H_{0}^{\prime \prime \prime}+\left(-\frac{1}{60} s^{2}-\frac{1}{120} s^{4}\right) Y\right] r^{-1} } \\
& +\frac{1}{8} s^{4} H_{0}^{\prime \prime 2} r^{-2}+\left(-\frac{9}{4} s^{2}+\frac{21}{8} s^{4}\right) H_{0}^{\prime} H_{0}^{\prime \prime} r^{-3}+\left[\frac{1}{4} s^{4} H_{0} H_{0}^{\prime \prime}+\left(\frac{75}{16} s^{2}-\frac{175}{32} s^{4}\right) H_{0}^{\prime 2}\right] r^{-4} \\
& +\left(-\frac{27}{8} s^{2}+\frac{69}{16} s^{4}\right) H_{0}^{\prime} H_{0} r^{-5}+\left(-\frac{7}{4} s^{2}+\frac{15}{8} s^{4}\right) H_{0}^{2} r^{-6} \\
B & {\left[\left(\frac{3}{20} s^{2}-\frac{7}{40} s^{4}\right) H_{0}^{\prime} H_{0}^{\mathrm{IV}}+\left(\frac{3}{5} s^{2}-\frac{9}{20} s^{4}\right) H_{0}^{\prime \prime} H_{0}^{\prime \prime \prime}+\left(\frac{1}{60} s^{2}+\frac{1}{120} s^{4}\right) Y\right] r^{-1} } \\
& +\frac{1}{8} s^{4} H_{0}^{\prime \prime 2} r^{-2}+\left(\frac{9}{4} s^{2}-\frac{21}{8} s^{4}\right) H_{0}^{\prime} H_{0}^{\prime \prime} r^{-3}+\left[\frac{1}{4} s^{4} H_{0} H_{0}^{\prime \prime}+\left(-\frac{75}{16} s^{2}+\frac{175}{32} s^{4}\right) H_{0}^{\prime 2}\right] r^{-4} \\
& +\left(\frac{27}{8} s^{2}-\frac{69}{16} s^{4}\right) H_{0}^{\prime} H_{0} r^{-5}+\left(\frac{7}{4} s^{2}-\frac{13}{8} s^{4}\right) H_{0}^{2} r^{-6} \\
\stackrel{(22)}{C} &
\end{aligned}
$$


where $s \equiv \sin \theta$ and $c \equiv \cos \theta$. By a coordinate transformation equivalent to (9.13) in [1], it can be shown that the above (22) solution describes, after the vibration, energy flowing inward equal to

$$
E_{\text {in }}=\frac{M^{2} Q^{2}}{30} \int_{u_{2}}^{\infty} H_{0}^{\prime \prime \prime 2} d v .
$$

\section{Appendix 2}

We consider here the ratio of energies transmitted inward by the tail and outward by the primary quadrupole oscillation discussed in Section 7 .

We now write the energies in units of customary dimensions (ucd) and denote them by $W_{\text {out }}$ and $W_{\text {in }}$. From (12)

$$
W_{\text {out }}=\frac{G Q^{2}}{30 c^{5}} \int_{u_{1}}^{u_{2}} \dddot{h}^{2} d u .
$$

To express $W_{i n}$ we first note that the dimensions of $H$ are (time $)^{-1} \times($ dimensions of $h$ ), so in ucd $H_{0}$ becomes

$$
\frac{2}{c} \int_{-\infty}^{u} \frac{h(\xi) d \xi}{(v-\xi)^{2}}
$$

We must also remember that $M$ must be replaced by $G m / c^{2}$, where $m$ is the central mass in ucd. Using this we have from (29)

$$
W_{i n}=\frac{G}{c^{5}}\left(\frac{G m}{c^{2}}\right)^{2} \frac{Q^{2}}{30} \frac{1}{c^{2}} \int_{u_{2}}^{\infty} H_{0}^{\prime \prime \prime 2} d v .
$$

Eqn (35) follows from (37) and (38) if we put $u_{1}=-1, u_{2}=1$.

\section{REFERENCES}

[1] Bonnor W B and Rotenberg M A 1966 Proc. R. Soc. A289 247

[2] Couch W E, Torrence R J, Janis A I and Newman E T 1968 J. Math. Phys. 9484

[3] Hunter A J and Rotenberg M A 1969 J. Phys. A (Gen. Phys.) 234

[4] Blanchet L and Schäfer G 1993 Class. Quantum Grav. 102699

[5] Blanchet L and Sathyaprakash B S 1994 Class. Quantum Grav. 112807

[6] Abrahams A M and Evans C R 1992 Phys. Rev. D46 R4117

[7] Abrahams A M and Evans C R 1993 Phys. Rev. Lett. 702980

[8] Kundu P K 1990 Proc. R. Soc. A431 337

[9] Price R H and Pullin J 1992 Phys. Rev. D46 2497

[10] Kozameh C, Newman E T and Rovelli C 1991 Phys. Rev. D44 551

[11] Price R H, Pullin J and Kundu P K 1993 Phys. Rev. Lett. 701572

[12] Bondi H, van der Burg M G J and Metzner A W K 1962 Proc. R. Soc. 
A269 21

[13] Bonnor W B 1974 Ondes et Radiations Gravitationelles (Paris:CNRS) p73

[14] Blanchet L and Damour T 1992 Phys. Rev. D46 4304

[15] Blanchet L 1997 Phys. Rev. D55 714 